\title{
Towards uncertainty mapping in air-quality modelling and assessment
}

\section{Bruce Denby*, Agnes Dudek and Sam Erik Walker}

Norwegian Institute for Air Research (NILU),

P.O. Box 100, 2027 Kjeller, Norway

E-mail: bruce.denby@nilu.no

E-mail: Agnes.Dudek@dnv.com

E-mail: sew@nilu.no

${ }^{*}$ Corresponding author

\section{Ana Margarida Costa and Alexandra Monteiro}

CESAM \& Department of Environment and Planning,

University of Aveiro,

3810-193 Aveiro, Portugal

E-mail: amcosta@ua.pt

E-mail: alexandra@dao.ua.pt

\section{Sef van den Elshout}

DCMR Environmental Protection Agency Rijnmond, P.O. Box 8433100 AV, Schiedam, The Netherlands

E-mail: sef.vandenelshout@dcmr.nl

\section{Bernard Fisher}

Risk and Forecasting Science,

Environment Agency, Kings Meadow House,

Kings Meadow Road, Reading RG1 8DQ, UK

E-mail: bernard.fisher@environment-agency.gov.uk

\begin{abstract}
The aim of this paper is to promote the use of uncertainty mapping when spatial assessments of air quality are made. A large number of air quality maps are produced for scientific and policy purposes but rarely are corresponding maps of their uncertainty included. The need for such maps and the methods to produce them are described. Several uncertainty parameters are discussed but it is recommended to use the probability density function as the basis of the uncertainty estimates. Several examples are provided discussing indicative uncertainty, ensemble methods, comparisons with observations, spatial representativeness, uncertainty in exceedances and probability of exceedance.
\end{abstract}

Keywords: air quality; assessment; modelling; uncertainty; data assimilation; Bayesian; probability; exceedance; mapping. 
Reference to this paper should be made as follows: Denby, B., Dudek, A., Walker, S.E., Costa, A.M., Monteiro, A., van den Elshout, S. and Fisher, B. (2011) 'Towards uncertainty mapping in air-quality modelling and assessment', Int. J. Environment and Pollution, Vol. 44, Nos. 1/2/3/4, pp.14-23.

Biographical notes: Bruce Denby is a Senior Scientist at NILU and has a background in mathematics, physics, meteorology and numerical methods for fluid dynamic modelling. He currently applies his expertise in air quality modelling, uncertainty assessment, exposure assessment, data assimilation and spatial statistical methods. He has a Doctoral Degree in Meteorology and Atmospheric Physics with emphasis on boundary layer turbulence processes and surface energy balance for glaciological and climatological studies (Utrecht, 2001). He has worked at the University of New South Wales (Australia), Utrecht University (The Netherlands), Cambridge University (United Kingdom) and at NILU.

Agnes Dudek in Aero-Mechanical Engineering from the University of Strathclyde, UK. She took her PhD at the Environmental Optic Research laboratory of Strathclyde University Applied Physics Department. In 2004, she was a post-doctoral scientist in development of high-resolution mesoscale numerical weather prediction model at the Norwegian Meteorological Institute of Norway. She joined NILU's Urban Environment and Industry Department in August 2005 where she worked in urban air quality modelling and was involved in model development of NILU's air quality information system AirQUIS. She has experience in air pollution, meteorology, remote sensing and GIS technology. She is currently employed as project manager in the Climate Change Services at DNV, Norway.

Sam Erik Walker has a BSc in Computer Science (Mathematics, Statistics and Numerical Analysis) from the University of Oslo. He has long experience in developing air pollution dispersion models and using numerical and statistical methods for analysing observations and model results, including techniques of data assimilation. He is the author of several of the present dispersion models at NILU, including the urban air pollution dispersion model EPISODE, which is part of NILUs AirQUIS system. He also works on data assimilation methodologies and techniques for application in air pollution modelling.

Ana Margarida Costa has a $\mathrm{PhD}$ in Sciences Applied to the Environment at the University of Aveiro. She is a Researcher at the R\&D Unit CESAM (Centre for Environmental and Marine Studies) of the University of Aveiro, and carries work in the field of traffic air pollutants dispersion in urban areas through the development and application of numerical modelling tools. Her current research interests also include the assessment of the outdoor air pollutants transport to indoors and human exposure to air pollution. She is the author of more than 65 scientific publications, including nine peer-reviewed papers in scientific journals, 20 papers in International Symposia with referee, and the participation in five book chapters.

Alexandra Monteiro is currently a Researcher and an Invited Professor at the Department of Environment of the University of Aveiro. She has the $\mathrm{PhD}$ in Sciences Applied to the Environment at the University of Aveiro, a Master of Science Degree in Atmospheric Pollution and a Degree in Chemical Engineering. She is part of the Research Group on Emissions, Modelling and Climate Change (GEMAC). Her research interests include regional and urban air quality, air quality forecasting, air pollution (emissions and modelling), forest fire emissions and pollution health effects. 
Sef van den Elshout is a senior staff member in the air quality unit of the Environmental Protection Agency (since 2001) in the Rotterdam port-industrial area. He is responsible for monitoring and modelling air quality for compliance checking, environmental licensing and managing air quality in spatial development. Before 2001 he worked in regional development and environmental planning in various African countries.

Bernard Fisher is an environmental modeller with more than 30 years research experience of air quality modelling. He joined the Environment Agency in 2000. Since 1994-2000, he was Professor of Environmental Modelling at the University of Greenwich and has published over 100 papers. Previously, he worked in the power industry and has experience of air quality management, dispersion modelling, the long-range transport of air pollutants and acid rain. $\mathrm{He}$ is a member of the UK Air Quality Expert Group, chairman of the Institute for Air Quality Management and is on the Council of the Institution of Environmental Sciences.

\section{Introduction and motivation}

Air quality assessment using monitoring or modelling is mandatory in all European member states through directives (EC, 1999, 2002). Inherent in every air quality assessment is uncertainty. When monitoring data is used, there is uncertainty in the instrumentation but most importantly in the spatial representativeness, since monitoring is limited to a few points in space, leading to a large uncertainty in the concentrations in the areas between the monitoring stations. This is one of the reasons modelling is used to assess air quality, as it provides information concerning the spatial distribution of the pollutants, as well as the ability to predict air quality when observations are not available. Model predictions are also uncertain in both space and time. For this reason, it is essential to assess the uncertainty of the models, not only at points in space but also spatially, for any air quality assessment.

Model uncertainty is due to errors in model formulation and input data as well as the natural spatial and temporal variability of the concentrations. Due to the finite temporal and spatial scales that models work on, these last two sources of uncertainty will always exist even with the 'best' models.

Model uncertainty is often described as model error at monitoring sites only, using a variety of parameters (e.g., Chang and Hanna, 2004). Uncertainty is rarely displayed as maps, however some examples do exist; Van de Kassteele and Velders (2006), Horálek et al. (2007) and Lindley and Walsh (2005) show maps of uncertainty (Standard Deviation (SD)) based on geo-statistical methods, Fuentes and Raftery (2005) present maps of bias (BIAS) and SD using a Bayesian approach and Rodriguez et al. (2007) display maps of SD based on Monte Carlo simulations.

It seems only reasonable that whenever air quality maps are shown that information on their uncertainty is also provided and preferably also as maps. The spatial uncertainty can give useful information on the quality of the model calculations and on the optimal placement of monitoring sites. It can also aid in establishing uncertainties when data assimilation methods are applied. When air quality assessment is made for risk assessment purposes, such maps provide valuable information for the responsible authorities that can aid in the decision-making process. 
This paper identifies methods for producing uncertainty maps for use in air quality assessment. It first discusses available uncertainty parameters that may be used to communicate the uncertainty and second the methods for their spatial calculation. Because of the variety of methods used for producing spatial assessments, the methods used to calculate the uncertainty also differ. For this reason, it is essential to find a characteristic parameter so that comparisons of methods and their uncertainties can be usefully made.

\section{Uncertainty indicators to be used for mapping}

There are a number of statistical parameters used for the assessment of model uncertainty that may also be appropriate for the spatial mapping of uncertainty. These include directive-related error indicators (EC, 1999) such as Relative Percentile Error (RPE), but also more standard statistical parameters such as SD, Root Mean Square Error (RMSE), BIAS and correlation $\left(r^{2}\right)$, see Borrego et al. (2007). These metrics have been mainly applied and developed for comparison with monitoring data on an individual basis and not for use in a spatial concept of uncertainty.

Approaches using Probability Distribution Functions (PDFs) can be used for uncertainty mapping. However, it is difficult to display spatio-temporal PDFs for a two-dimensional field, see Pebesma et al. (2007). PDFs can be simplified by using analytical functions, such as normal or log-normal distributions, that are described by a limited set of parameters. A normally distributed PDF can be defined by the parameters of BIAS and SD. If the assumption of normal distributions is not met, then other distributions, such as log-normal or more general discretised distributions, can be used. The SD uncertainty concept may still be applied for these though the description is more complex. An alternative is to define the appropriate minimum and maximum percentile bands and use their separation to define the uncertainty. For the case of a normally distributed PDF, the separation of the $2.5 \%$ and $97.5 \%$ percentile band is simply four times the SD.

Figure 1 Left: PDF of daily mean $\mathrm{PM}_{10}$ concentrations calculated by the unified EMEP model using observations from Airbase (2003). Note the strong negative bias of the model. The continuous grey line is a log-normal fit to the data. Right: PDF of hourly mean $\mathrm{NO}_{2}$ concentrations at a local traffic site in Oslo as calculated using the EPISODE model. Note the two modes of the model, a result of uncertainty in the wind direction as calculated by the model. Only observations with a concentration $>10 \mu \mathrm{gm}^{-3}$ are used
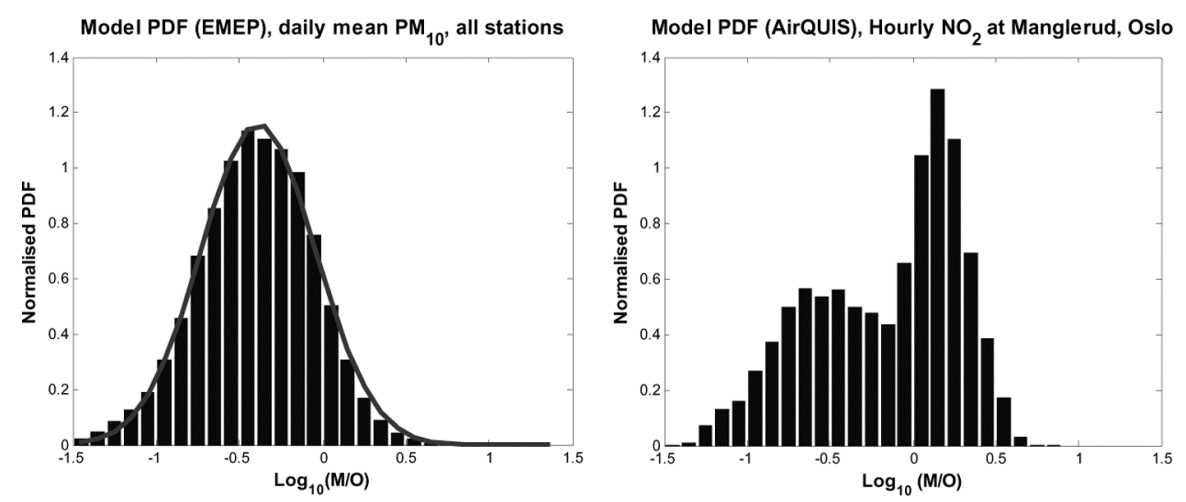
One method for constructing a PDF, based on observations, is by calculating the ratio $\mathrm{M} / \mathrm{O}$ for corresponding model and observational points. By binning these values in discrete steps, the PDF can then be constructed, see Figure 1 for two examples. When using this approach, a minimum threshold value should be used for the monitoring data.

\subsection{Relative maximum or percentile error}

The present European legislation defines the Modelling Quality Objectives as an acceptability measure to guarantee good model performance and reliable modelling results for decision-makers. When model uncertainty is required for directive purposes, then the most relevant parameter is RPE, evaluated at monitoring sites (see Air4EU, 2007; M2). However, as previously described, this may not be a good indicator of spatial uncertainty.

\subsection{Standard deviation}

It is recommended to use uncertainty parameters related to the SD. SD is useful because it resembles other uncertainty parameters that may be calculated such as RMSE, kriging variance, SD resulting from Monte Carlo ensembles, or covariance matrices required for data assimilation techniques. The SD of a PDF can also be calculated for any discrete distribution. However, SD is only a good uncertainty indicator when the system is unbiased. It is thus also useful to indicate uncertainty in maps using BIAS as well.

\subsection{Bias}

BIAS represents the long-term (e.g., annual) mean difference between models and observations over a defined spatial region. Usually, BIAS is calculated at observational points assuming the observations to be unbiased. When the model is representative of a larger area than the observations, then BIAS may be incorrectly attributed to the model owing to the spatial variability of the concentrations. BIAS thus has a spatial context, being dependent on the scale, and this should be considered when it is assessed. However, given a set of representative observations, it is possible to map the BIAS on the scale of interest, see Section 3.1, or preferably to remove the spatial component of the BIAS by using methods such as regression or kriging techniques.

\section{Methods for calculating the spatial distribution of uncertainty}

\subsection{Spatial representation of uncertainty calculated at observational sites}

When model assessment is carried out at spatially distributed monitoring sites, statistical parameters of model error can be determined at these points. This error may be represented as individual points on the map or alternatively, when there is a sufficient density of stations and the pollutant varies on scales larger than the typical distance between stations, it may be interpolated to represent the spatial uncertainty of the model. 
Such parameters may include the SD, RMSE or any other error indicator. This method of representing uncertainty is straightforward but the question of its validity when interpolated in space arises owing to the question of spatial representativeness. Figure 2 provides an example of such an application where RPE is used to assess model error for ozone calculations in and around Berlin. It is represented as both points and as an interpolated field (Air4EU, 2007; D7.1.14).

Figure 2 Mapping of Relative Percentile Error (RPE) of an $\mathrm{O}_{3}$ simulation (concerning the 26th maximum $8 \mathrm{~h}$ running average percentile) with the RGC model over Berlin (Air4EU, 2007; D7.1.14). Left: RPE at the 49 individual monitoring sites is represented by the size of the circles. Right: RPE is interpolated using kriging in the same domain
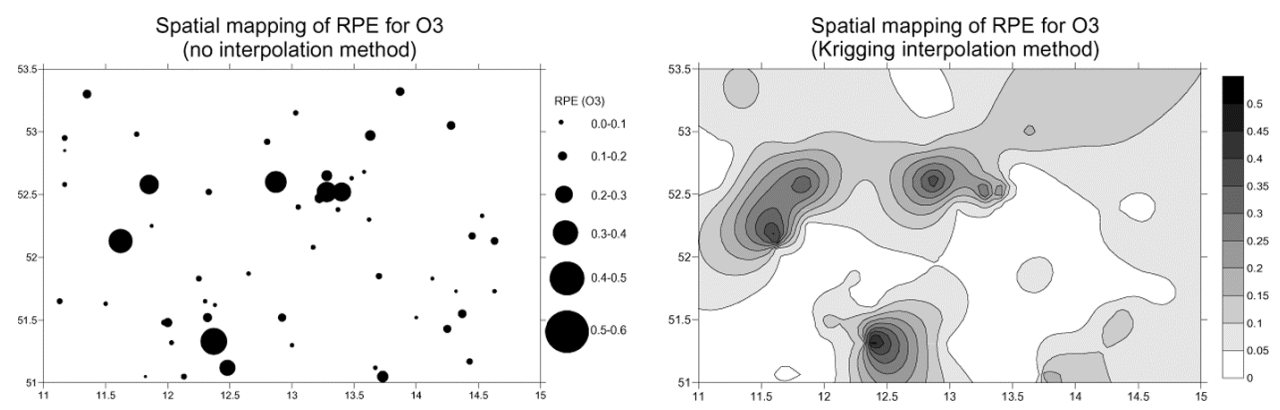

\subsection{Indicative uncertainty as a function of model concentration}

This type of uncertainty map treats the uncertainty, using $\mathrm{SD}(\sigma)$ as the uncertainty parameter, as a function of the absolute uncertainty $\sigma_{A}$, the relative uncertainty $\sigma_{R}$ and the model concentration $M(x, y)$ in the following form

$$
\sigma_{M}(x, y)=\sqrt{\sigma_{A}^{2}+\sigma_{R}^{2} M(x, y)^{2}} .
$$

This provides a general structure for calculating uncertainty as a function of model concentrations, and thus spatially. The estimated values of $\sigma_{R}$ and $\sigma_{A}$ may be calculated in various ways, e.g., based on experience with the model, based on the normalised RMSE (Figure 3), based on the SD of PDFs or, when a sufficient amount of data is available, by fitting equation (1) to the SD of the model error when it is expressed as a function of model concentration $M(x, y)$. This last method may be achieved by binning the observed concentrations in discrete intervals, e.g., $10 \mu \mathrm{g} . \mathrm{m}^{-3}$ bins, and calculating the $\mathrm{SD}$ of the model error for each of these bins.

There are a number of reasons why this method may be appropriate. First, when there are few observational data available, a best estimate of $\sigma_{R}$ and $\sigma_{A}$ can be made; second, there are a number of situations where model uncertainty can be directly coupled to emission uncertainty and direct scaling using $\sigma_{R}$ is appropriate; third, uncertainties in model formulation that apply generally to the modelling domain often affect the calculations in either an absolute or a relative fashion. 
Figure 3 Example of an assessment (left) and uncertainty map (right) of the annual mean $\mathrm{NO}_{2}$ concentrations in Prague, 2003 (Air4EU, 2007; D7.1.6). The uncertainty map is constructed using $\sigma_{R}$ based on the normalised RMSE of 12 stations where $\sigma_{R}=27 \%$ and $\sigma_{A}=0$
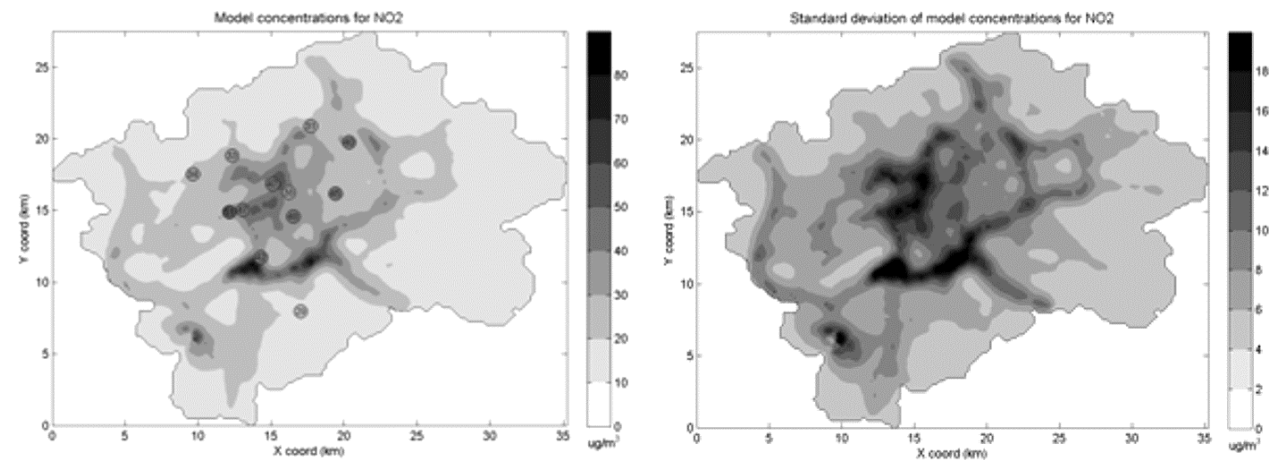

\subsection{Maps based on data assimilation, statistical and kriging variance}

When data assimilation or other statistically based methods are used, for example linear regression analysis, kriging and residual kriging, variational methods or ensemble techniques such as Ensemble Kalman filters, estimates of the variance/covariance are required for the assimilation technique and can be used in the uncertainty maps.

Though these methods produce estimates of SD, it represents different elements of the uncertainty. When ensemble methods are used, then the SD conveys information about uncertainties related to the perturbed parameters used to create the ensemble, not the predictive uncertainty of the model. Geo-statistical methods such as kriging represent uncertainties that are directly related to the variance of the observations. These are based on a functional relationship between variance and the distance from a monitoring station assuming reasonably homogeneous fields relative to the distance between the stations.

\subsection{Mapping the uncertainty in exceedances}

For some applications, the Number of Exceedances (NOE) above some threshold value for a short averaging period is required, e.g., daily mean $\mathrm{PM}_{10}$ concentrations. In principle, the NOE at any point in space can be calculated as the sum of the POEs occurring for each averaging period. This can be assessed by integration of the PDF at that point in space and for that period. However, this is not suitable for models containing representativeness errors and unknown BIAS. POE is more sensitive to uncertainty in BIAS than it is to the uncertainty in an unbiased model. Then, to represent the uncertainty in the NOE field, a pragmatic approach is recommended. The uncertainty in the NOE is calculated by use of the annual mean SD percentile band, i.e., by adding and subtracting the annual mean SD from the daily mean value $( \pm \sigma)$, which reflects the model and representativeness BIAS. The uncertainty in NOE days can be interpreted as being the maximum deviation, in number of days, from the $\pm \sigma$ calculations (Air4EU, 2007; D7.1.13). An example is provided in Figure 4 showing the results of such an uncertainty assessment for the $\mathrm{NOE}$ days of $\mathrm{PM}_{10}$ in Europe. 
Figure 4 European map of the assimilated, using residual kriging methods, (left) and uncertainty (right) fields for the number of daily mean exceedances of $\mathrm{PM}_{10}$ above a threshold of $50 \mu \mathrm{g} . \mathrm{m}^{-3}, 2003$. Using this methodology uncertainty is lowest, but not zero, in the regions with good observational coverage (Air4EU, 2007; D7.1.13)
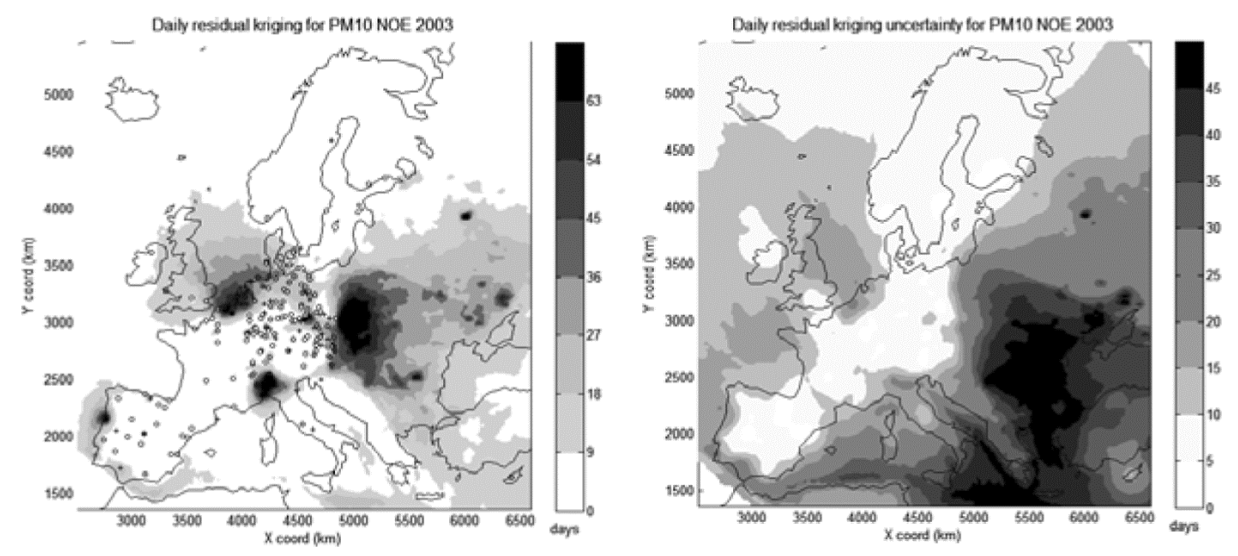

\subsection{Model uncertainty using ensemble simulations}

Another method for estimating uncertainty is to carry out ensemble runs by perturbing model parameters and input data (within their uncertainty) to obtain estimates of the intrinsic model uncertainty (e.g., Rodriguez et al., 2007). This can be carried out using various Monte Carlo methods where the PDFs of the model parameters are used as prior distributions for the ensembles. This will give a spread of model results that can be mapped. It will not provide information on BIAS unless direct comparisons are made with observations. As a special case of the Monte Carlo methods, some uncertainties can be analysed in a simpler framework. If input parameters, such as emissions, can be simply described by a normal distribution and the model is linear, then the uncertainty can be directly calculated by summing the uncertainties from the various emission sources.

Similar to Monte Carlo simulations within a model, simulations using an ensemble of models (e.g., van Loon et al., 2007) can provide spatially distributed estimates of uncertainty, though this has yet to be carried out in such studies.

\subsection{Probability of exceedance}

Given both concentration and uncertainty fields, as PDFs or in terms of SD, it is possible to determine the POE, given some limit value, by integration of the PDF. POE includes both calculated concentrations and uncertainty information in the one parameter and may be useful in applications for risk assessment. POE is not an uncertainty parameter itself and a POE map does not show to what extent the map is determined by the model calculations or the model uncertainty. 


\section{Conclusions}

In this preliminary paper, a number of methods for producing uncertainty maps have been briefly described and some examples given. It is recommended that SD be the major indicative uncertainty parameter in the uncertainty maps and that whatever be the assessment method applied, an uncertainty assessment of the derived air quality map should always be provided in some form. It is not recommended to spatially interpolate model errors when the distance between monitoring stations is larger than their representative scale. If BIAS is known, then it should be indicated and if possible removed from the assessment maps.

More work is still required to define methods for the spatial assessment of uncertainty and there are a number of challenges that still need to be addressed. Convincing the air-quality modelling community and other stakeholders and decision-makers to include, or request, uncertainty in their maps is one of these. Cooperation between atmospheric modellers and spatial statistical groups is also needed. Finally, and perhaps, most challenging of all is the reformulation of directives and other legislation to properly include aspects of uncertainty.

\section{Acknowledgement}

This research was funded by the EU FP6 project Air4EU. Contract No. (503596)

\section{References}

Air4EU (2007) Air4EU Deliverable Reports, URL: http://www.air4eu.nl/reports_products.html

Borrego, C., Monteiro, A., Ferreira, J., Miranda, A.I., Costa, A.M. and Sousa, M. (2007) 'Modeling uncertainty estimation procedures for air quality assessment', in Topçu, S., Yardim, M.F., Bayram, A., Elbir, T. and Kahya, C. (Eds.): Proceedings of the 3rd International Symposium on Air Quality Management at Urban, Regional and Global Scales (AQM), 26-30 September 2005, Istanbul, Turkey, Vol. I, pp.210-219.

Chang, J.C. and Hanna, S.R. (2004) 'Air quality model performance evaluation', Meteorol. Atmos. Phys., Vol. 87, pp.167-196.

EC (1999) First Daughter Directive, Council Directive 1999/30/EC, relating to limit values for sulphur dioxide, nitrogen dioxide and oxides of nitrogen, particulate matter, and lead in ambient, OJ L 163, 29 June, pp.41-60, URL: http://eur-lex.europa.eu/LexUriServ/site/en/oj/ 1999/1_163/1_16319990629en00410060.pdf

EC (2002) Third Daughter Directive, Council Directive 2002/3/EC, relating to ozone in ambient air, OJ L 67, 09 March, pp.14-30. URL: http://eur-lex.europa.eu/pri/en/oj/dat/2002/1_067/ 1_06720020309en00140030.pdf

Fuentes, M. and Raftery, A.E. (2005) 'Model evaluation and spatial interpolation by Bayesian combination of observations with outputs from numerical models', Biometrics, Vol. 66, pp.36-45.

Horàlek, J., Denby, B., de Smet, P., de Leeuw, F., Kurfurst, P., Swart, R. and van Noije, T. (2007), Spatial Mapping of Air Quality for European Scale Assessment, ETC/ACC Technical paper 2006/6. URL: http://air-climate.eionet.europa.eu/reports/ETCACC_TechnPaper_2006_6_Spat $\mathrm{AQ}$.

Lindley, S.J. and Walsh, T. (2005) 'Inter-comparison of interpolated background nitrogen dioxide concentrations across greater Manchester', Atmos. Environ., UK, Vol. 39, pp.2709-2724. 
Pebesma, E.J., de Jong, K. and Briggs, D.J. (2007) 'Visualising uncertain spatial and spatio-temporal data under different scenarios: an air quality example', International Journal of GIS (Special Issue in Honour of the Contribution of Peter Burrough to Geographical Information Science), Vol. 21, No. 5, pp.515-527.

Rodriguez, M.A., Brouwer, J., Samuelsen, G.S. and Dabdub, D. (2007) 'Air quality impacts of distributed power generation in the South coast air basin of California 2: model uncertainty and sensitivity analysis', Atmos. Environ., Vol. 41, pp.5618-5635.

Van de Kassteele, J. and Velders, G.J.M. (2006) 'Uncertainty assessment of local $\mathrm{NO}^{2}$ concentrations derived from error-in-variable external drift kriging and its relationship to the 2010 air quality standard', Atmos. Environ., Vol. 40, No. 14, pp.2583-2595.

van Loon, M., Vautard, R., Schaap, M., Bergström, R., Bessagnet, B., Brandt, J., Builtjes, P.J.H., Christensen, J.H., Cuvelier, C., Graff, A., Jonson, J.E., Krol, M., Langner, J., Robertj, P., Rouil, L., Stern, R., Tarrasón, L., Thunis, P., Vignati, E., White, L. and Wind, P. (2007) 'Evaluation of long-term ozone simulations from seven regional air quality models and their ensemble', Atmos. Environ., Vol. 41, No. 10, pp.2083-2097. 